\title{
A BAYESIAN FRAMEWORK FOR UNCERTAINTY FORMULATION OF ENGINEERING DESIGN PROCESS
}

\author{
M. RAJABALINEJAD ${ }^{\dagger}$, C. SPITAS \\ Faculty of Industrial Design Engineering, TUDelft
}

2628CE Delft, the Netherlands

\begin{abstract}
Uncertainties in the design process are investigated in this paper. A formal Bayesian method is presented for designers to quantify uncertainties in design process. The uncertainties are implemented in a decision support system that plays a key role in design of complex projects where a large and multidisciplinary team of engineers are involved. The proposed method produces the probability distribution function of the model score or uncertainty. Two example applications for design and empirical demonstrate application of the method.
\end{abstract}

\section{Introduction}

In this paper, a formal approach is provided to formulate the uncertainties of the design process by integration of the prior information and data. These are important elements in the design process since designers start the design with a program of requirements which include a large amount of uncertainty [1, 2]. Then, data and information are used to reduce uncertainty and to improve the prior information of the designer. As a result, implementation of a technique that can integrate the prior information of the designer and the accumulated data to assess the overall uncertainty of the problem is of great value to design process in system analysis and product development. For this purpose, we use a Bayesian method based on the global uncertainty concept [3, 4]. This robust concept helps us to unify different uncertainties into one. In other words, the uncertainties sourced from experts, designers, users and clients are unified using

\footnotetext{
† Assistant Professor, ${ }^{\text {a }}$ M.Rajabalinejad@tudelft.nl
} 
the concept of the global uncertainty. These results are used for a decision support tool which leads a designer to robust product design [5].

The use of graphs and drawings is preferred to effectively communicate with users. Because designers are usually strong in visual communication, we embrace visual communication with designers to present the uncertainties or confidence visually. Having this said, we used a simple table for this purpose with a scale factor ranging from 0 to 100 . This is shown in Figure 1. The values of 0 and 100 represent two extreme limits respectively, for no chance of acceptance, and absolutely satisfied criterion for a certain concept. Figure 1(b) represents a concept that satisfies a certain criterion between $20 \%$ and $80 \%$.

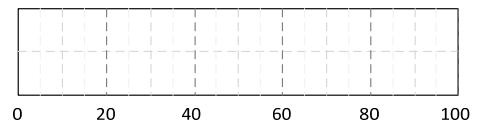

(a)

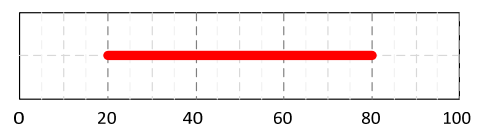

(b)

Figure 1. (a) Survey form for an expert to present his opinion within a confidence interval. (b) The confidence interval presented by the expert.

Assume that there are a number of criteria $i$ for a few given concepts. Given these, we wish to make a rational decision by choosing between the competing concepts. The expert opinion for each concept is given by a set of random variables with Gaussian distribution, $s=\left\{s^{(1)}, s^{(2)}, \ldots, s^{(1)}\right\}$. On the basis of the rational frame work, we consider the first moment of these random variables as the scoring function $[4,6]$. Assuming different weight factor for each criteria, we use the following equation to rank the candidate concepts and select the concept with the highest score:

$$
\mu=\frac{1}{\sum_{k=1}^{i} \lambda_{k}}\left[\sum_{k=1}^{i} \lambda_{k} \int \frac{1}{\sqrt{2 \pi} \sigma^{(k)}} \exp \left(\frac{\left(x-\mu\left(s^{(k)}\right)\right)^{2}}{2\left(\sigma^{(k)}\right)^{2}}\right) x d x\right],
$$

where $\mu$ is the given score (rank) to the $\mathrm{j}$-th concept, $i$ is the total number of criteria, $\lambda_{i}$ is the weight factor of criterion $i$.

\section{Mathematical Formulation}

Consider a model score function $\mu$ that we wish to estimate based on some prior information, $I$, and data, $D$. The prior information is the result of the designer's knowledge, while the data comes out of the interview with stakeholders, experts, users, etc. Let $P(\mu \mid D, I)$ be the univariate probability density function (pdf) representing the model score as a random variable. The data $D$ and prior information $I$ are obtained from the designers, experts or interviews (surveys). 
Let $\sigma$ be the global uncertainty which relates all local uncertainties. The global uncertainty $\sigma$ (e.g., global standard deviation) was first used in [7-9] to define a nuisance random variable. With marginalization and the global uncertainty, we can write

$$
P(\mu \mid D, I)=\int P(\mu, \sigma \mid D, I) d \sigma .
$$

By application of Bayes Theorem, we find

$$
P(\mu \mid D, I)=\int P(\mu \mid \sigma, D, I) P(\sigma \mid D, I) d \sigma .
$$

We need to define the first term $P(\mu \mid \sigma, D, I)$ in the RHS of Equation (3). The value of error with a zero mean can be positive or negative and its unknown variance is $\sigma^{2}$. We use index $\mathrm{j}$ for this to associate it with the designer, and the designer's error by $e_{j}$. We use the Gaussian density function to model the error in a standard error form [10]. We define the Gaussian error as

$$
P\left(e_{j} \mid \sigma_{j}\right)=\frac{1}{\sqrt{2 \pi} \sigma_{j}} \exp \left\{-\frac{1}{2 \sigma_{j}^{2}} e_{j}^{2}\right\},
$$

where $e_{j}$ is the error and $\sigma_{j}^{2}$ is the unknown variance. This error represents the difference between the model score $\mu$ and the designer's estimate $\hat{\mu}_{j}$, $e_{j}=\mu-\hat{\mu}_{j}$. By making the change of variable from $e_{j}$ to $\mu$, we obtain the following multivariate pdf for the pixels $\mu$ as:

$$
P\left(\mu \mid \sigma_{j}, D, I\right)=\frac{1}{\sqrt{2 \pi} \sigma_{j}} \exp \left\{-\frac{1}{2 \sigma_{j}^{2}}\left(\mu-\hat{\mu}_{j}\right)^{2}\right\} .
$$

Following the approach used in [7], we can associate the global and local uncertainties by defining

$$
\sigma_{j}=\alpha_{j} \sigma
$$

where $\alpha_{j}=\sqrt{\left|\mu-\hat{\mu}_{j}\right|}$. We apply the Gaussian distribution in Equation (4) to determine the error of the $\mathrm{i}$-th measured data point $e_{i}$ with unknown variance $\sigma_{i}^{2}$ and a mean value $\hat{u}_{i}$ obtained from the collected data, $D$. For $n$ data points, the error of the model score for any of the observed data points may be defined as:

$$
e_{i}=\mu-d_{i},
$$


where $i=1, \ldots, n$. Assuming the error in Equation (7) has a zero mean (e.g., the error may have positive or negative values) with an unknown variance $\sigma_{i}^{2}$, it can be concluded that

$$
P\left(\sigma \mid \mu, d_{1}, \ldots, d_{n}\right)=\frac{1}{(2 \pi)^{n / 2}\left(B \sigma^{n}\right)} \exp \left\{-\frac{1}{2 \sigma^{2}} \sum_{i=1}^{n} \frac{\left(d_{i}-\mu\right)^{2}}{\alpha_{i}^{2}}\right\},
$$

where $B$ is

$$
B=\alpha_{1} \cdots \alpha_{n} .
$$

Multiplication of Equations (5) and (8) according to Equation (3) and integration over $\sigma$ gives the posterior $P(\mu \mid D, I)$ as

$$
P(\mu \mid D, I) \propto \frac{1}{B^{\prime}}\left[\frac{\frac{\left(\mu-\hat{\mu}_{j}\right)^{2}}{\alpha_{j}^{2}}}{\sum_{i=1}^{n}\left\{\frac{\left(d_{i}-\mu\right)^{2}}{\alpha_{i}^{2}}\right\}}+1\right]^{-\frac{1}{2} n},
$$

where $B^{\prime}=B \alpha_{j}$. The numerical algorithm best for implementing these formulas is summarized next.

\section{Application example: COLD FACTS Project}

The Cold Facts is a program of the Dutch World Wide Fund (WWF Netherlands) established or climate change studies in the Polar Regions to build a reliable and lightweight weather station deployed in North Pole. The design team for this project is composed of 15 designers from different faculties of TUDelft. Decision-making in the design process was a challenge at the managerial level because of the experience level and the diversity of designers' knowledge. This challenge was explicitly addressed through a series of reports and presentations.

To clarify the implementation aspect of our method, we selected three criteria for selecting the final concept. These criteria were: reliability, aesthetics, and light weight. We considered these to be the most important requirements of the model. In the next step, Concept 1 and Concept 2 were presented to an expert, asking for his expert opinion. The designer's prior knowledge for either of the concepts is the same as given in Figure 2. For the demonstration purpose, we present here the results of integration of three different experts with a designer's opinion on either of the concepts. These are shown in Figure 4, representing the probability density function (pdf) of the model score. Having calculated the first moment of these pdfs, the best concept can now be chosen. 


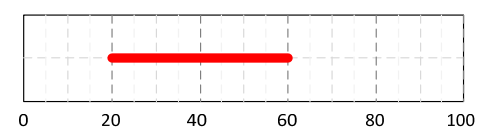

Figure 2. The designer's opinion on the reliability of either of the concepts.

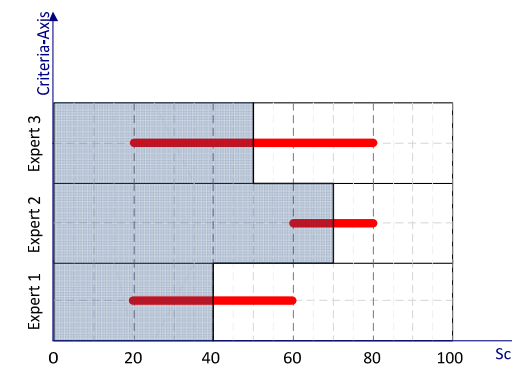

(a)

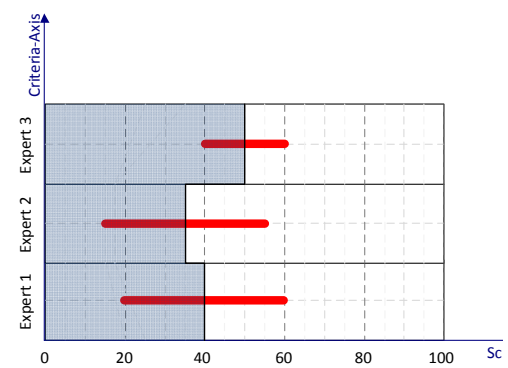

(b)

Figure 3. (a) The reliability of Concept 1 is scored by three different experts. (b) The reliability of Concept 2 is scored by three different experts.

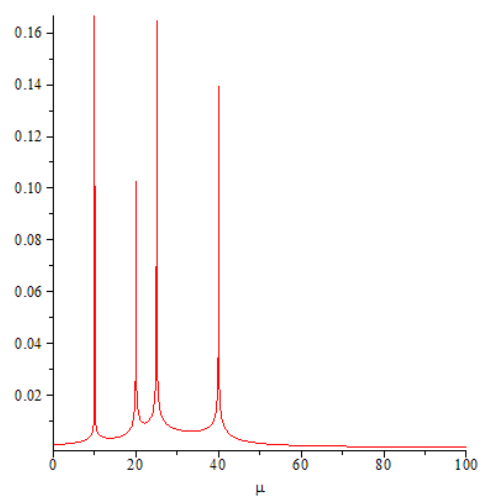

(a)

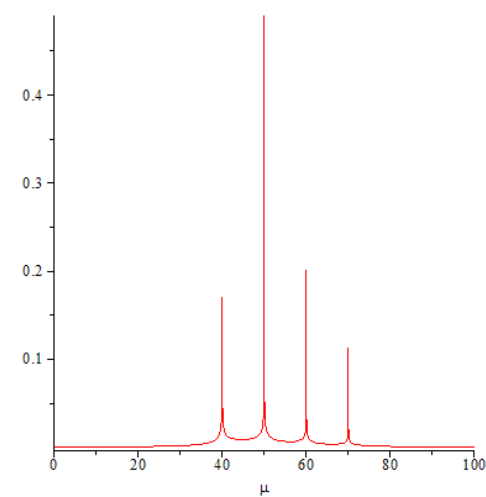

(b)

Figure 4. This figure presents two different probability density functions assigned to the concepts for the Cold Facts project.

\section{CONCLUSIONS}

A detailed description of the theoretical formulation and numerical implementation for a Bayesian framework of a probabilistic approach and its integration with the design process are provided in this paper . The proposed hybrid approach is based on the Bayesian interpolation method. We present a methodology that enables us to formulate the uncertainties of different elements in design process and integrate the prior information with the data. This is 
important in the design process because at each stage, there is a prior information, which needs to be integrated to the prior information in order to reduce the uncertainty and make more educated decisions.

The application of graphical tool is demonstrated here in a numerical example for the Cold Facts project. This project was chosen because of an interdisciplinary team of designers and experts who had different level of experience and expertise. The proposed and applied method relies on experts' and designers' knowledge, and integrates these uncertainties into the decisionmaking process.

\section{References}

1. Rajabalinejad, M. and C. Spitas, Incorporating Uncertainty into the Design Management Process. Design Management Journal, 2011: p. 11.

2. Spitas, C., Analysis of systematic engineering design paradigms in industrial practice: Scaled experiments. Journal of Engineering Design, 2011. 22(7): p. 447-465.

3. Rajabalinejad, M. and T. Mahdi, The inclusive and simplified forms of Bayesian interpolation for general and monotonic models using Gaussian and Generalized Beta distributions with application to Monte Carlo simulations. Natural Hazards, 2010. 55(1): p. 29.

4. Rajabalinejad, M., Reliability Methods for Finite Element Models. I ed2009, Amsterdam, the Netherlands: IOS Press.

5. Du, X. and W. Chen, Efficient uncertainty analysis methods for multidisciplinary robust design. AIAA journal, 2002. 40(3): p. 545581.

6. Cover, T.M. and J.A. Thomas, Elements of information theory2004: Wiley Online Library.

7. Rajabalinejad, M., Reliability Methods for Finite Element Models. I ed2009, Amsterdam, the Netherlands: IOS Press.

8. Rajabalinejad, M., P. van Gelder, and N. van Erp, Application of Bayesian Interpolation in Monte Carlo Simulation, in Safety, Reliability and Risk Analysis (ESREL)2008, Taylor and Francis Group, London, UK. p. 705-713.

9. Rajabalinejad, M., Bayesian Monte Carlo method. Reliability Engineering and System Safety, 2010. 95(10): p. 1050-1060.

10. Jaynes, E.T., Probability theory, the logic of science. 2003. 